\title{
À propos du subjonctif en français : une révision expérimentale de l'effet de l'affaiblis- sement de l'obviation
}

\author{
Sebastian Buchczyk ${ }^{1}$, et Ingo Feldhausen ${ }^{1, *}$ \\ ${ }^{1}$ Goethe-Universität Frankfurt, Institut für Romanische Sprachen und Literaturen, Norbert-Wollheim- \\ Platz 1, 60629 Frankfurt am Main, Allemagne
}

\begin{abstract}
Résumé. Les données de l'effet d'affaiblissement de l'obviation (ou encore référence disjointe $d u$ subjonctif) dans la littérature linguistique sont soit fondées sur l'intuition des auteurs soit issues de publications d'autres chercheurs, bien que cet effet soit connu depuis longtemps et bien qu'il fasse partie de plusieurs modélisations théoriques. En conséquence, aucune réponse de nature empirique/expérimentale a été offerte à la question «A quel point les affirmations portant sur l'effet d'affaiblissement de l'obviation sont-elles solides ? ». Cet article se fixe en conséquence un double objectif : (i) mettre en œuvre un jugement de grammaticalité afin de répondre expérimentalement à la question précédente, et (ii) proposer une analyse syntaxique provisoire des résultats obtenus. Ici, nous étudions six facteurs qui - selon Ruwet (1984/1991) - déclenchent l'affaiblissement. Les résultats montrent que seul un facteur (la coordination) déclenche l'affaiblissement. En conséquence, l'appareil théorique permettant de modéliser l'affaiblissement peut être réduit radicalement, et nous proposons des pistes de modélisation syntaxique n'intégrant que le facteur de coordination.
\end{abstract}

\begin{abstract}
On the subjunctive in French: experimentally revisiting the effect of obviation weakening. Even though the weakening of the subjunctive disjoint reference effect, also known as obviation, plays an important role in the research of subjunctives in (non-)Romance languages, to the best of our knowledge it has never been verified experimentally. The goal of our paper is twofold: (i) to test how native speakers of French evaluate sentences displaying factors that should weaken obviation using a grammatical judgement task, and (ii) to propose an initial novel framework for a possible syntactic approach to address the attested patterns. Our results show that only one out of six factors described in Ruwet $(1984 / 1991)$ weakens obviation in French, namely Coordination. Since this factor is syntactic rather than semantic, the theoretical framework can be simplified, and we thus propose a syntactic analysis based on Farkas' (1992) competition approach and Zhang's (2010) analysis of coordination structures.
\end{abstract}




\section{Introduction}

Dans cet article, nous souhaitons souligner l'importance d'une approche empirique/ expérimentale de la recherche linguistique à l'aide d'un aspect spécifique des structures présentant un subjonctif en français. Plus concrètement, nous nous concentrons sur le phénomène de l'obviation et sur son affaiblissement. L'obviation est caractérisée par l'impossibilité d'une coréférence entre le sujet de l'énoncé matrice et le sujet de l'énoncé subordonné qui est au mode subjonctif (1a). Pour qu'une coréférence soit possible, le locuteur doit utiliser l'infinitif dans l'énoncé subordonné (1b); dans ce dernier cas, le sujet de l'énoncé subordonné est tacite (indiqué par " _ »). La coréférence entre les deux sujets dans (1b) est indiquée par l'index 1.

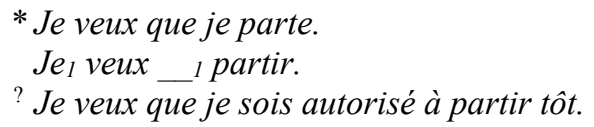

Dans un travail novateur, Ruwet (1984/1991) a montré qu'il était toutefois possible d'avoir une coréférence entre les deux sujets dans des structures comme (1a) - voir (1c). Selon Ruwet (1984/1991), il y a certaines conditions/facteurs qui rendent une coréférence plus acceptable (ce qui est indiqué par un «? » dans (1c)) - voir la section 2. Pour Ruwet (1984/1991) le phénomène en (1c) illustre un affaiblissement de l'obviation : l'énoncé n'est plus agrammatical, mais il n'est pas à 100\% acceptable non plus.

Depuis Ruwet (1984/1991), l'affaiblissement de l'obviation occupe une part importante de la recherche et de la théorie linguistique conduite sur le subjonctif dans une grande variété des langues (voir la section 2). Toutefois, jusqu'à présent, l'affaiblissement n'a jamais été vérifié expérimentalement (voir la section 3) - à notre connaissance. C'est la raison pour laquelle nous avons conduit une étude expérimentale. Nous voulions savoir jusqu'à quel point les affirmations de Ruwet (1984/1991) portant sur l'effet d'affaiblissement de l'obviation en français sont solides. Dans la section 4, nous décrivons notre expérience, qui est une tâche de jugement de grammaticalité, et nous présentons les résultats. Par la suite, nous proposons une approche théorique permettant de modéliser les résultats (voir la section 5). Nous soutiendrons qu'il est possible de défendre un traitement économique de l'obviation, puisque seul un des six facteurs de Ruwet (1984/1991) présente un affaiblissement - un résultat obtenu grâce à l'approche expérimentale.

\section{Le subjonctif, l'obviation et son affaiblissement}

\subsection{Le subjonctif et le phénomène de l'obviation}

Le subjonctif est un mode du français (voir De Mulder 2010, Le Goffic 1993, Mosegaard Hansen 2016 : \#5.4., Riegel et al. 2014 : \#X.2.2, e.a.). Au contraire du mode de l'indicatif, exprimant un fait réel au moment où l'on rapporte (2a), le subjonctif est typiquement décrit comme le mode qui révèle le caractère d'un évènement ou état qui n'est qu'imaginé, désiré ou nécessaire. À l'aide du subjonctif, le locuteur peut également envisager un point de vue, un souhait, un ordre, etc. (2b).

Dites-leur que Pierre viendra ce soir. (= Indicatif : fait réel)

Dites à Pierre qu'il vienne ce soir. （= Subjonctif : souhait) 
De surcroît, le subjonctif exprime une référence disjointe entre le sujet de la phrase subordonnée et le sujet de la phrase matrice. Dans l'exemple (3), les sujets dans les énoncés subordonné et matrice se réfèrent à des individus différents, d'un côté Pierre et de l'autre côté l'interlocuteur $t u$. Les crochets en (3) indiquent les limites de l'énoncé subordonné.

Pierre veut [que tu partes].

Cette référence disjointe entre les deux sujets quand l'énoncé subordonné est au subjonctif est connue comme obviation ou en anglais subjunctive disjoint reference effect (SDRE) (voir Ruwet 1984/1991, Picallo 1985, Kempchinsky 1987, 2009, Farkas 1992, Luján 1999, Costantini 2005, 2009, 2016, Szucsich 2009, Feldhausen 2007, 2010 : ch.4, Romero Mérida 2013, e.a.). Par conséquent, sont agrammaticaux les énoncés complexes avec un subjonctif enchâssé au sein desquels les deux sujets sont coréférents (4).

* Je veux que je parte.

* Tu veux que tu partes.

Les énoncés de type (4a,b) amènent des chercheurs (comme Ruwet 1984/1991, Luján 1999, Costantini 2005, e.a.) à l'observation que dans une structure complexe, à savoir un énoncé inséré dans un autre, qui a un complément au subjonctif, la coréférence entre les deux sujets est interdite, ou bien agrammaticale.

Néanmoins, pour exprimer une coréférence entre les deux sujets dans une structure complexe, il est possible d'employer l'infinitif (Farkas 1992, Schlenker 2005). Le pronom phonologiquement nul dans l'énoncé subordonné (marqué par " _ ») se réfère au sujet de la phrase principale (marqué par l'index ${ }_{1}$ ), voir (5).

$$
\begin{aligned}
& \mathrm{Je}_{1} \text { veux _l partir. } \\
& \mathrm{Tu}_{1} \text { veux_l partir. }
\end{aligned}
$$

\subsection{L'effet de l'affaiblissement de l'obviation}

La définition du phénomène de l'obviation ne permet pas que les sujets de la phrase subordonnée et de la phrase matrices soient coréférents. Toutefois, Ruwet (1991) prétend qu'il existe malgré tout des possibilités d'affaiblir l'obviation. Voir les exemples $(6 \mathrm{~b}, \mathrm{c})$ comparés à (6a) (=4a).

* Je veux que je parte.

? Je veux que je puisse partir.

? Je veux que je sois autorisé à partir tôt.

Ruwet (1991) remarque que selon son intuition, les phrases $(6 \mathrm{~b}, \mathrm{c})$ sont assez acceptables, malgré la coréférence entre les sujets, et plus acceptable que (6a), qui est clairement inacceptable.

Les effets de l'affaiblissement de l'obviation sont aussi décrits pour d'autres langues romanes, comme l'italien (7a ; Costantini 2009 : 79), le catalan (7b ; Costantini 2009 : 36), l'espagnol (7c; Quer 2006: 664) et le portugais (7d; Raposo 1985 : 86). Dans chaque exemple, on peut voir qu'il existe toujours une relation coréférente entre les sujets de la proposition subordonnée et de la principale, et que cette relation n'est pas complètement exclue. Au sens strict, selon les auteurs, les cas de l'espagnol et du portugais démontrent que la coréférence peut même être parfaitement acceptable. 
it. ? $\quad$ pro 1 Voglio assolutamente [que io 1 sia partito entro dieci minuti.]

cat. ?'En Joan ${ }_{1}$ està sorprès [que pro ${ }_{1}$ hagi fet molts errors.]

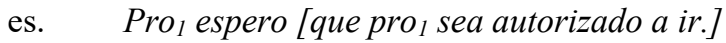

b.

pt. $\quad O$ Manel $_{1}$ deseja [que pro $_{1}$ seja admitido no concurso.]

c.

d.

\subsubsection{Les facteurs de l'affaiblissement selon Ruwet (1991)}

En se concentrant sur le français, Ruwet (1991) discute plusieurs facteurs d'affaiblissement ; nous en examinerons six. Ces six facteurs sont fondés sur deux causes, à savoir (i) la réduction de l'agentivité du sujet de l'énoncé subordonné, et (ii) l'expression d'une certaine distance entre le souhait et l'achèvement de l'action exprimée par le locuteur (voir Ruwet 1991: 20-21). Par conséquent, si ces deux causes s'appliquent à un énoncé au subjonctif, l'obviation est affaiblie, et la coréférence sera plus acceptable. Il est à noter que les jugements des énoncés dans cette section sont uniquement fondés sur l'intuition de Ruwet (1991). Dans ce qui suit, nous présentons les six facteurs qui nous analyserons dans notre étude expérimentale. Il s'agit :

1. du passif

2. du passé composé /du temps périphrastique

3. de la négation

4. des verbes de modalité

5. des verbes psychologiques

6. de la coordination

Le premier facteur que nous décrirons est le passif. Dans les énoncés (8a) et (9a), on observe les formes verbales du passif dans l'énoncé subordonné. Les formes correspondantes à l'infinitif sont proposées en $(8 b)$ et $(9 b)$. Bien que Ruwet (1991:20) remarque que les énoncés $(8 \mathrm{a})$ et $(9 \mathrm{a})$ sont pires que leurs pendants à l'infinitif, il les met à côté exemples $(4 a, b)$, montrant qu'ils ne sont pas si mauvais qu'ils pourraient être considérés comme inacceptables. Selon lui, la raison pour laquelle $(8 \mathrm{a}, \mathrm{b})$ sont malgré tout plus acceptables est assez simple: le passif, par définition, n'exprime pas une action réalisée par le sujet lui-même; en d'autres termes, c'est à cause de la réduction de l'agentivité par la diathèse du passif que (8a) et (9a) sont rendus plus acceptables. Ruwet (1991 : 20) parle d'un détour de l'achèvement de l'action du locuteur qui dépend de la volonté de quelqu'un d'autre. De surcroît, le contenu sémantique du verbe pourrait jouer un rôle important pour l'acceptabilité de la phrase : l'emploi du verbe autoriser indique que le pouvoir d'une tierce personne surpasse celui du locuteur, tandis que enterrer suggère que le locuteur ne sera plus présent pour s'assurer de l'achèvement de sa volonté.

?Je veux que je sois autorisé à partir demain. Je veux être autorisé à partir demain.

?Je veux que je sois enterré dans mon village natal. Je veux être enterré dans mon village natal.

En ce qui concerne le deuxième facteur, le passé composé, l'énoncé subordonné est de nouveau au centre de notre attention - voir (10). Pour Ruwet (1991 : 23), cette forme de la flexion verbale temporelle dans l'énoncé subordonné représente un exemple typique d'expression de la distance entre la volonté et l'achèvement d'une action. C'est pourquoi, selon lui, il suffit de mettre l'énoncé enchâssé au passé composé, qui met en valeur l'accomplissement d'une action aussi bien en (10a) que (10b). Ce changement dans la morphosyntaxe verbale rend à lui seul les énoncés grammaticaux. 
Je veux (absolument) que je sois parti dans dix minutes. Ah! Je voudrais que je sois déjà parti.

La négation représente le troisième facteur. Ici, nous regardons l'énoncé matrice dans lequel nous trouvons le verbe nié avec la construction « ne ... pas », suivi par l'énoncé subordonné au subjonctif (11a,b). Selon Ruwet (1991 : 29), cette négation du verbe matrice influe sur l'affaiblissement de telle sorte que si l'énoncé matrice est mis à la forme négative et que l'énoncé subordonné contient également une expression d'apparence négative, l'énoncé entier sera plus acceptable. L'explication d'une plus haute acceptabilité est que le locuteur exprime qu'il ne veut pas que cet évènement se répète. Ceci est valable pour les deux cas $(11 a, b)$. En effet, le locuteur n'est pas en mesure d'avoir une (grande) influence sur ce qui se passera à l'avenir. Donc, Ruwet (1991 : 30) en tire la conclusion que l'affaiblissement est lié à la deuxième cause, à savoir la distance entre la volonté et la mise en œuvre de l'action.

\footnotetext{
?Je ne veux pas que je rate une occasion pareille.

?Je ne veux pas que je me trompe de clé (encore).
}

Les verbes de modalité représentent le quatrième facteur. Selon Ruwet (1991), la coréférence avec un verbe conjugué dans l'énoncé subordonné sera plus acceptable si ce verbe est un verbe de modalité comme pouvoir en (12a). Une fois de plus, c'est l'énoncé subordonné qui est modifié. Ruwet (1991 : 21) remarque que (12a) sera moins acceptable que son pendant à l'infinitif (12b). Toutefois, avec un verbe de modalité dans l'énoncé subordonné, l'énoncé entier est plus acceptable que ceux en (4). Néanmoins, il soutient que l'acceptabilité dépend de la situation. Par exemple, si l'énoncé (12a) était exprimé dans une situation dans laquelle un homme d'affaires demande à son secrétaire de faire une réservation pour une place en avion, l'énoncé serait encore plus acceptable (Ruwet 1991 : 21).

\section{? Je veux que je puisse partir dès demain. Je veux pouvoir partir dès demain.}

Le cinquième facteur concerne les verbes psychologiques que l'on peut retrouver dans les énoncés subordonnés - voir (13b) et (14b). Ce qui est caractéristique des verbes psychologiques selon Ruwet, c'est qu'ils oscillent entre une interprétation agentive et nonagentive. Pour Ruwet (1991 : 27), ils représentent donc un problème intéressant. C'est pourquoi il remarque que les énoncés (13b) et (14b) sont ambigus à cause de leur interprétation agentive ou non-agentive (1991: 27). En revanche, les exemples (13a) et (14a) sont strictement non-agentifs. En raison de l'ambigüité en (13b) et (14b), l'interprétation non-agentive en interaction avec l'obviation permet la tendance du locuteur à voir une deuxième instance de soi-même, comme s'il se voyait de l'extérieur. Donc, cette deuxième instance établit encore une fois la distance entre le souhait et l'achèvement de l'action.

?Je veux que j'amuse ces enfants.

Je veux amuser ces enfants.

?? Je veux que je sois étonné.

Je veux qu'on m'étonne. 
La coordination est le dernier facteur qui introduit la possibilité d'établir un contraste entre le locuteur et l'interlocuteur, ayant pour résultat un énoncé acceptable. En (15) et (16), nous voyons des énoncés subordonnés avec deux conjoints, à savoir une forme verbale avec « tu » (ou « il »), et l'autre avec « je ». En (15a), c'est le pronom du premier conjoint qui est coréférent avec le sujet de l'énoncé matrice. Dans les autres exemples, $(15 b)$ et $(16 a, b)$, c'est le pronom du deuxième conjoint qui est coréférent et, donc, il y a toujours un conjoint avec un pronom non-coréférent qui est posé entre les deux pronoms coréférents. Cette distance entre les deux éléments coréférents est pertinente pour Ruwet (1991:24) : (15a) est inacceptable à cause du sujet coréférent dans la phrase subordonnée qui se trouve dans le premier conjoint. Toutefois, $(15 \mathrm{~b})$ est plus acceptable parce que le sujet de l'énoncé subordonné est à distance du sujet de l'énoncé matrice (Ruwet 1991 : 24). De plus, en combinaison avec les verbes de modalité comme pouvoir dans (16a), ou le passif dans (16b), l'énoncé entier devient parfaitement grammatical selon Ruwet (1991 : 24). L'effet des facteurs, donc, peut-être plus fort si les facteurs s'additionnent.

* Je veux que je parte et que tu restes.

? Je veux que tu partes et que je reste.

Je veux que tu partes et que je puisse rester.

Je veux qu'il soit vaincu et que je sois vainqueur.

\section{Contexte et motivation de l'étude}

L'obviation et l'effet de l'affaiblissement de l'obviation sont connus depuis longtemps, et il existe une abondante littérature qui les discute en détail pour des langues différentes, comme le français, l'espagnol, le catalan, l'italien, mais aussi le hongrois, le russe ou le polonais (p. ex. Bouchard 1982, 1983, Ruwet 1984/1991, Raposo 1985, Picallo 1985, Suñer 1986, Farkas 1992, Costantini 2005, 2009, 2013, 2016, Schlenker 2005, Quer 2006, 2017, Feldhausen 2007, 2010: ch.4, Szucsich 2009). Il existe différentes propositions de modélisation théorique de cet effet. Selon Costantini (2005) et d'autres, il est possible de les catégoriser dans deux grandes démarches distinctes : une partie des modélisations est fondée sur la théorie du liage (p. ex. Picallo 1985, Raposo 1985, Kempchinsky 1987, Rizzi 1991, Manzini 2000), et l'autre fondée sur la démarche de compétition (p. ex. Bouchard 1982, 1983, Farkas 1992, Schlenker 2005). Nous n'entrerons pas ici dans les détails de ces modélisations. Ce qui est important pour nous est la source des données montrant l'effet de l'affaiblissement de l'obviation : une étude de la littérature montre que les données sont soit fondées sur l'intuition des auteurs (p. ex. Ruwet 1984/1991, Suñer 1986, Costantini 2009), soit issues de publications d'autres chercheurs (p. ex. Farkas 1992, Quer 2006, 2017). Notre examen de la littérature révèle qu'il n'existe pas de preuve expérimentale de l'effet de l'affaiblissement de l'obviation. ${ }^{i}$ En conséquence, notre question de recherche est celle qui est développée en (17) :

A quel point les affirmations portant sur

l'effet d'affaiblissement de l'obviation sont-elles solides?

Pour répondre à cette question, il nous faut une (plus) vaste étude empirique/ expérimentale sur les effets de l'affaiblissement s'appuyant sur des tâches de jugements de grammaticalité (angl. grammaticality judgment tasks) ou des études de linguistique de corpus. Dans ce qui suit, nous nous appuierons sur le jugement de grammaticalité. L'étude de Ruwet (1984/1991) étant fondamentale pour la recherche conduite sur l'effet d'affaiblissement, nous nous concentrerons ici sur ses facteurs et sur le français. 


\section{Etude expérimentale}

\subsection{Méthode}

\subsubsection{Participants}

Notre groupe test comprend 88 locuteurs/locutrices natifs/-ves du français ayant entre 18 et 76 ans, inscrits à l'université, exerçant une profession ou en retraite. Seuls quatre participants ont un diplôme inférieur au baccalauréat. Leur niveau de langue en français a été vérifié grâce à une brève phase de test au sein du questionnaire.

\subsubsection{Matériel et conception}

Une tâche de jugement de grammaticalité (angl. grammaticality judgement task - GJT) a été employée (voir Featherson 2006, Sprouse 2018). Nous avons préparé huit énoncés test pour chacun des six facteurs (voir la section 2.2.1), ce qui correspond à 48 énoncés test au total. La même quantité d'énoncés de remplissage a été retenue; en conséquence, la quantité totale d'énoncés évalués par chaque participant est de 96 énoncés. Tous les énoncés étaient pseudo-randomisés (pas plus de 2 énoncés consécutifs pour un même facteur). Chaque énoncé était affiché séparément, suivi par une échelle d'évaluation du type Likert-7 (Schütze \& Sprouse 2013) au-dessous de l'énoncé test. Le participant était libre d'utiliser l'échelle d'évaluation dans son intégralité, choisissant n'importe lequel des sept points d'évaluation, limités par les pôles « grammatical», " agrammatical » et « ni l'un ni l'autre » au milieu de 'échelle - voir la figure 1.

Tu veux que tu partes et que je reste.

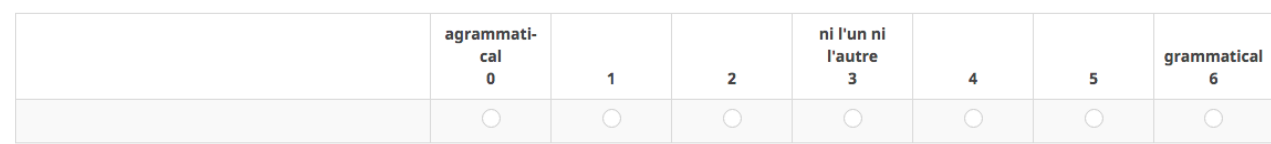

Fig. 1. Exemple d'énoncé test

Chaque participant a évalué l'ensemble des 96 énoncés, mais l'ordre des énoncés variait : on distinguait deux blocs, A et B, qui comprenaient des énoncés par groupes de 48 énoncés pseudo-randomisés. Un groupe a été affecté à l'ordre $\mathrm{AB}$, un autre à l'ordre $\mathrm{BA}$. En combinant les blocs des ordres $\mathrm{AB}$ et $\mathrm{BA}$, nous employions deux versions du sondage avec les mêmes énoncés test. L'ordre interne des blocs, c'est-à-dire l'ordre des énoncés dans les blocs A et B, n'était pas affecté.

\subsubsection{Procédure}

Les participants étaient testés individuellement en remplissant un sondage en ligne sans supervision de l'examinateur. Nous avons utilisé l'outil/le software "LimeSurvey». Une séance durait entre 15 et 20 minutes. Elle comprenait d'abord la complétion d'une phase de test, ou familiarisation, afin de vérifier la compétence linguistique des participants, puis la phase de sondage dans laquelle les participants devaient évaluer les 96 énoncés du test. À la fin du sondage, les participants devaient remplir un bref questionnaire social.

\subsection{Résultats}

La figure 2 montre au moyen d'une statistique descriptive les évaluations de chacun des six facteurs. Il est immédiatement évident qu'il y a une grande différence entre le facteur 
Coordination, avec une valeur de moyenne de 4,24 d'un côté, et les cinq autres facteurs, avec des valeurs de moyenne allant de 1,40 à 1,73 de l'autre côté. Le facteur Coordination est évalué comme étant plutôt grammatical alors que les autres facteurs sont évalués comme étant plutôt agrammaticaux. La déviation standard est comprise entre 1,53 et 1,68 pour les six facteurs. La figure 3 montre la distribution des évaluations pour les facteurs PsychVerbs et Coordination. La distribution des quatre autres facteurs est similaire à celle des PsychVerbs. La figure 3 montre une variation dans les décisions des participants, mais les évaluations du facteur PsychVerbs sont plutôt basses, alors que les évaluations du facteur Coordination pointent plutôt vers le haut.

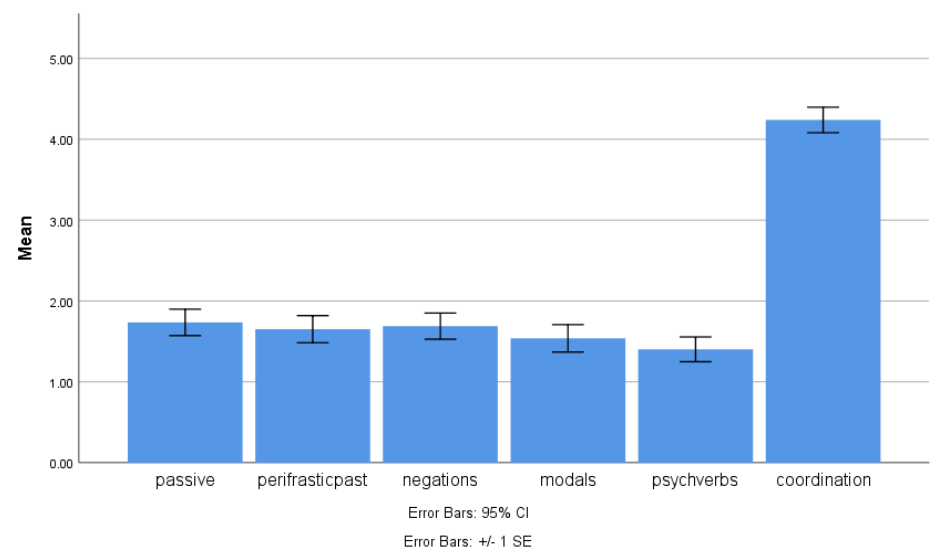

Fig. 2. Evaluation des six facteurs listés par Ruwet (1991).
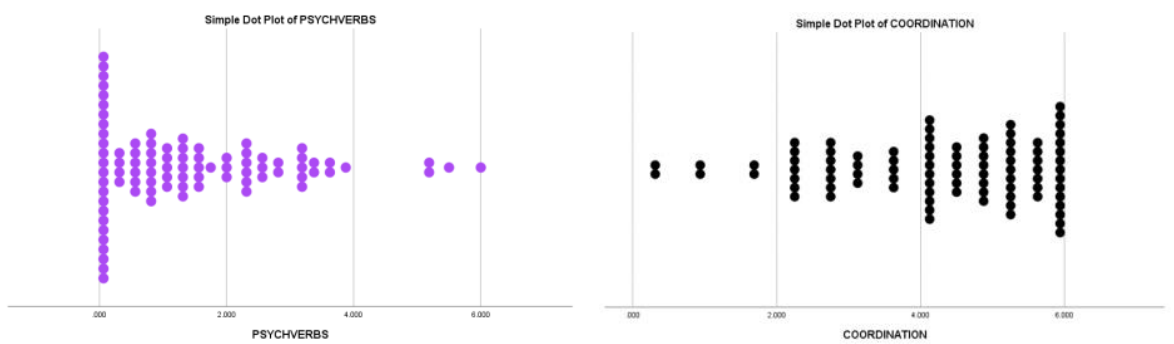

Fig. 3. Évaluation individuelle des facteurs PsychVerbs (à gauche) et Coordination.

Pour mieux juger les évaluations des facteurs de l'affaiblissement en figure 2, nous présentons dans le tableau 1 quelques valeurs des énoncés agrammaticaux, grammaticaux et les contreparties des phrases test du facteur Coordination. Ces énoncés ont fait partie de nos phrases de remplissage.

Nous avons utilisé comme énoncés agrammaticaux des énoncés tels que Filler 2 et Filler 42. Leur moyenne est de 1,36. Cette moyenne est plutôt proche de celle de nos phrases test pour les cinq facteurs hors Coordination. En conséquence, nous concluons que nos phrases tests sont agrammaticales et que les cinq facteurs en question ne jouent pas un rôle important dans l'affaiblissement de l'obviation.

En revanche, nos énoncés grammaticaux (Filler 13 et Filler 21) sont jugés avec une valeur moyenne très haute $(5,59)$, qui est assez proche de la valeur 6 de notre échelle. La valeur du facteur Coordination $(4,24)$ n'est pas aussi haute que celle de nos énoncés grammaticaux. Pourtant, le facteur Coordination est jugé comme étant assez bon par les participants. 
Notre caractérisation de la valeur de Coordination est soutenue par une comparaison avec les énoncés qui constituent la contrepartie agrammaticale. Alors que les sujets identiques dans nos énoncés test sont éloignés les uns des autres (JE veux que tu partes et que JE reste), ce n'est pas le cas de ceux des contreparties (JE veux que JE parte et que tu restes). Ces derniers sont jugés avec une valeur moyenne de 2,08. Ils sont, dès lors, jugés moins bons que nos phrases test, même s'ils sont un peu mieux évalués que les énoncés des cinq autres facteurs $(1,40-1,73)$.

Tableau 1. Evaluation des phases de remplissage sélectionnées.

\begin{tabular}{|c|c|c|}
\hline Valeur (moyenne) & Enoncé & Classification \\
\hline 1,26 & $\begin{array}{l}\text { *Tu veux que tu partes. } \\
\text { (Filler } 2 \text { ) }\end{array}$ & \multirow{2}{*}{ Agrammatical } \\
\hline 1,46 & $\begin{array}{l}\text { *Je trouve être génial. } \\
\text { (Filler } 42 \text { ) }\end{array}$ & \\
\hline 5,49 & $\begin{array}{l}\text { Hier, je suis sorti de ma maison très vite. } \\
\text { (Filler 13) }\end{array}$ & \multirow{2}{*}{ Grammatical } \\
\hline 5,68 & $\begin{array}{l}\text { Hier, tu es sorti de ta maison très vite. } \\
\text { (Filler } 21 \text { ) }\end{array}$ & \\
\hline 1,87 & $\begin{array}{l}\text { *Je veux que je parte et que tu restes. } \\
\text { (Filler } 7 \text { ) }\end{array}$ & \multirow{2}{*}{$\begin{array}{c}\text { Contrepartie } \\
\text { Coordination } \\
\text { (agrammatical) }\end{array}$} \\
\hline 2,29 & $\begin{array}{l}\text { *Tu veux que tu partes et que je reste. } \\
\text { (Filler } 18 \text { ) }\end{array}$ & \\
\hline
\end{tabular}

Dans notre expérience, nous avons par ailleurs demandé l'origine des locuteurs afin de déterminer s'il existe une différence dialectale dans les jugements (distribution de nos participants par région: Hauts-de-France: 27 ; Ille-de-France : 18; Auvergne-RhôneAlpes: 12 ; Autres : 31). Un test de Kruskal-Wallis, qui est non-paramétrique et qui s'utilise lorsque plus de deux groupes sont impliqués, montre qu'il n'y a pas de différence dialectale significative (Passive : $p=.412$; Temps périphrastique : $p=187$; Négation: $p=.068$; Verbes modaux : $p=.203$; Verbes psychologiques : $p=.207$; Coordination : $p=.223)$.

A la lumière de tout ce que nous venons de développer, nous concluons que seul le facteur de coordination montre un affaiblissement de l'obviation, alors que ce n'est pas le cas des autres facteurs. De plus, nous n'avons observé aucune variation dialectale. Par conséquent, une modélisation théorique de l'effet d'affaiblissement doit donc expliquer l'influence du facteur Coordination et peut ignorer les autres facteurs et la variation dialectale. Dans ce qui suit, nous proposons une telle modélisation.

\section{Modélisation théorique - une proposition}

Comme nous l'avons dit auparavant, il existe deux démarches théoriques pour l'obviation. Les deux types d'hypothèses qui ont été suivis jusqu'à présent sont (i) les démarches fondées sur la théorie du liage ${ }^{\mathrm{ii}}$ et (ii) les démarches de compétition. Ce sont ces dernières qui nous semblent pertinentes. Ici, nous proposons des idées pour une modélisation de l'effet de l'affaiblissement de l'obviation déclenché par le facteur de coordination fondée sur l'approche de Farkas (1992) combinée à l'analyse de coordination de Zhang (2010). Dans ce qui suit, nous présenterons notre analyse, après avoir présenté les idées de Farkas (1992) et de Zhang (2010).

Dans les démarches de compétition, les auteurs supposent qu'il existe une compétition entre les modes du subjonctif et de l'infinitif. L'infinitif (19) bloque le subjonctif (18) quand il y a coréférence entre le sujet de l'énoncé matrice et le sujet de l'énoncé 
subordonné. Les représentations de ces démarches sont construites sur l'idée de blocage d'Aronoff (1976), qui dit qu'une construction spécifique exclut une construction générale dans un contexte particulier. Ici, l'infinitif est plus spécifique que le subjonctif, puisqu'il reprend les propriétés référentielles d'un autre argument comme condition lexicale.

$$
\begin{array}{ll}
\text { Subjonctif (plus général) } & * \text { Je veux [ que je parte ]. } \\
\text { Infinitif (plus spécifique) } & \quad J e_{1} \text { veux [__ partir ]. }
\end{array}
$$

Farkas (1992), travaillant sur l'espagnol et le hongrois, crée deux généralisations sur l'apparition de l'obviation, que l'on a adopté puis adapté à notre modélisation : (i) il y a une référence disjointe entre le sujet matrice et le sujet subordonné quand ce dernier se voit assigné le rôle thématique d'agent, et (ii) l'obviation apparait dans des contextes de volonté et de désir.iii Dans les deux énoncés espagnols en (20) et (21) le verbe matrice est un verbe de désir: esperar 'espérer' (en accord avec la généralisation (ii)). En (20), le sujet subordonné se voit assigné le rôle thématique d'agent par le verbe subordonné ayudar 'aider'. En conséquence, il doit exister une référence disjointe entre les deux sujets. (20) est agrammatical, puisque les sujets sont coréférents. Cependant, le verbe subordonné ser autorizado 'être autorisé' en (21) est au passif et, en conséquence, n'assigne pas le rôle thématique d'agent au sujet subordonné. C'est la raison pour laquelle l'effet de l'affaiblissement apparaît, et la coréférence devient acceptable.

$$
\begin{array}{lll}
\text { Obviation } \quad \text { es. } & * \text { pro }_{1 \mathrm{sg}} \text { Espero [ que pro }{ }_{1 \mathrm{sg}} \text { le ayude en ir ]. } \\
& \text { 'J'espère l'aider à s'en aller.' } \\
\text { Affaiblissement } & \text { es. } \quad \text { pro }_{1 \mathrm{sg}} \text { Espero [ que } \text { pro }_{1 \mathrm{sg}} \underline{\text { sea autorizado a ir ]. }}
\end{array}
$$

Le point faible de cette démarche de Farkas (1992) est la modélisation de l'effet de l'affaiblissement par la coordination. L'énoncé en (22a) est agrammatical, alors qu'un changement d'ordre des conjoints conduit à un affaiblissement de l'obviation (22b). La raison pour laquelle on devrait avoir un changement de force agentive entre (22a) et (22b) n'est pas évidente - avec la coordination, il n'y a pas de réduction de la force agentive. Par conséquent, la généralisation (i) ne fonctionne pas : on ne devrait pas avoir un affaiblissement de l'obviation (22 - exemple de Ruwet (1991: 23: ex.48)).

\section{* Je veux [que je partes et que tu restes]. \\ ? Je veux [ que tu partes et que je reste].}

Pour résoudre cette difficulté, nous supposons avec Zhang (2010) que la localité/minimalité joue un rôle important. Zhang (2010) montre qu'elle est importante en ce qui concerne le comportement des conjoints dans des structures de coordination. Costantini (2009) montre qu'elle joue un rôle significatif dans l'occurrence de l'obviation au sein de doubles enchâssements. Pour notre analyse, nous allons mettre les deux faits en relation.

Zhang (2010 : 50-55) suppose que les conjoints sont positionnés dans le spécificateur et dans le complément d'une SX - (23). De plus, le spécificateur détermine la projection maximale de la coordination entière («category-sharing»; indiquée par le cercle) : la sélection $c$ de la préposition anglaise on 'sur' est remplie par le premier conjoint my assistance 'mon assistant' et non par le deuxième conjoint that he will be on time 'qu'il va être ponctuel' - voir (24).

[DP1 [DP1 conjoint 1] $\left[\mathrm{x}\right.$, [ $\mathrm{x}^{\circ}$ et] [DP2 conjoint 2]]] 
angl. You can depend on my assistance and that he will be on time.

* You can depend on that he will be on time.

b.

* You can depend on that he will be on time and my assistance.

Le premier conjoint dispose d'une relation plus proche avec le syntagme supérieur que le second. De façon similaire, on peut voir que la localité est aussi pertinente auprès de l'obviation. La relation est visible dans le cas d'un double enchâssement. L'obviation apparaît seulement entre le sujet subordonné et celui de la proposition immédiatement supérieure, mais pas entre $\mathrm{CP}_{2}$ et l'énoncé matrice - (25).

$$
\text { Jean } 1 \text { veut [CP1 qu'il } *_{1 / 2} \text { désire [CP2 } q u^{\prime} i_{l_{1 * 2}} \text { aime Marie]]. }
$$

En nous appuyant sur ces diverses idées, nous disposons maintenant des outils permettant de modéliser l'affaiblissement de l'obviation dans les structures de coordination - le seul facteur d'importance pour l'affaiblissement selon notre étude. L'effet de la coordination dans (22) peut être saisi par les hypothèses suivantes : (a) L'obviation apparait dans des contextes de volonté et de désir, (b) l'obviation apparaît seulement localement et (c) dans des structures de coordination, le premier conjoint joue un rôle particulier. Selon Zhang (2010), il remplit la propriété c-sélectionnelle du verbe matrice. Ici, seul le premier conjoint est pertinent pour la compétition entre l'infinitif et le subjonctif - voir (26).

Je veux...

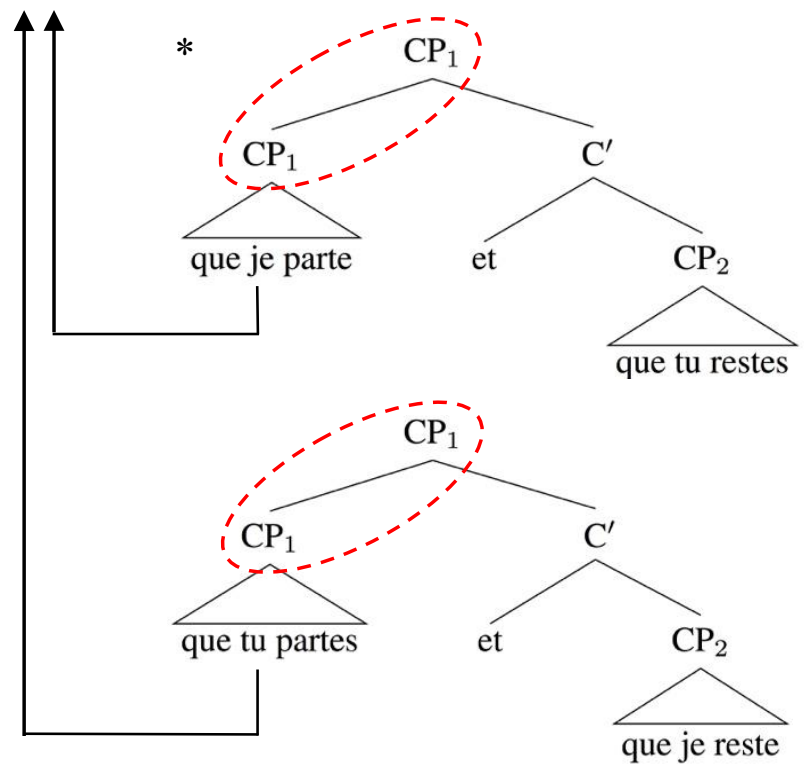

a.

Alors que la compétition existe dans (26a), ce n'est pas le cas en (26b). L'énoncé (26a) est agrammatical, puisque l'on doit utiliser la forme la plus spécifique (l'infinitif) pour la coréférence (c'est-à-dire: ?Je veux partir et que tu restes). En revanche, (26b) est grammatical, puisqu'il n'y a pas de forme plus spécifique. Pour se référer à une autre personne que le sujet de l'énoncé matrice, on ne peut pas utiliser l'infinitif : or, le subjonctif doit être utilisé. Le fait que le sujet du deuxième conjoint dans (26b) est coréférent avec le sujet de l'énoncé matrice peut être ignoré, puisque seul le premier conjoint joue un rôle pour la compétition. 
De plus, notre analyse est capable d'expliquer l'agrammaticalité des énoncés fondés sur les facteurs 1 à 5 de Ruwet (voir la section 2.2.1). Ici, il y a une compétition entre le subjonctif et l'infinitif - et le dernier bloque le premier. Dans notre analyse, l'agentivité ne joue pas un rôle (en contraste avec Farkas 1992) et, en conséquence, il n'y a aucun moyen (et pas de nécessité) de permettre un affaiblissement.

\section{Discussion et conclusion}

Dans cet article, nous avons souligné l'importance de la fondation empirique pour la recherche linguistique à l'aide d'un aspect spécifique des structures avec un subjonctif en français; plus concrètement, nous avons revisité le phénomène d'obviation et son affaiblissement.

Notre question de recherche (à quel point les affirmations portant sur l'effet d'affaiblissement de l'obviation sont-elles solides ?) a connu les réponses qui suivent. Nous avons montré des différences entre les affirmations sur l'effet de l'affaiblissement dans Ruwet (1984/1991) et nos résultats empiriques sur le français. Plus concrètement, notre étude empirique pilote a montré (a) que l'effet d'affaiblissement ne semble pas être réel, (b) que seules les structures avec coordination révèlent l'affaiblissement, (c) que le rôle de l'agent n'est pas pertinent, et (d) que la variation dialectale n'apparaît pas.

En conséquence, une démarche théorique fondée sur le rôle de l'agentivité pour modéliser l'affaiblissement n'est pas nécessaire - au moins pour le français. Ce qui est pertinent est la localité et des aspects structurels des énoncés; le phénomène semble être de nature syntaxique. Par conséquent, une réduction de l'effort théorique est possible, puisqu'une analyse théorique construite à l'interface syntaxe-sémantique n'est pas nécessaire (p. ex. les relations RESP(ONSABILITY) de Farkas (1992) ou attitude bearer de Costantini (2009)).

En accord avec cette idée, nous proposons une modélisation qui est construite sur la supposition d'une compétition entre l'infinitif (plus spécifique) et le subjonctif (non spécifique) - voir Farkas (1992) -, sur le rôle de la localité et le rôle central du premier conjoint (à partir des idées de Zhang 2010). L'avantage est clair: des preuves indépendantes existent pour la compétition et pour la localité dans les coordinations, et nous avons adapté ces idées à notre analyse. De plus, nous avons réduit l'effort théorique afin de modéliser le phénomène d'affaiblissement de l'obviation.

Pour poursuivre au-delà de notre étude pilote, que l'on a présentée dans cet article, nous pensons qu'il est important d'inclure plus d'énoncés contrôle qui doivent être (a)grammaticaux afin de mieux évaluer les valeurs des six facteurs qui ne sont ni clairement agrammaticaux (c'est-à-dire ayant une valeur 0) ni clairement grammaticaux (c'est-à-dire ayant une valeur 6). Ainsi, il sera possible de normaliser les valeurs, de les comparer et de les analyser statistiquement.

D’une manière générale, nous espérons que notre étude a démontré la contribution prometteuse d'une démarche expérimentale à la recherche théorique de phénomènes comme l'obviation - un phénomène qui est discuté principalement à partir de données fondées sur l'intuition. À côté d'une étude expérimentale comme la nôtre, il serait intéressant de s'approcher de l'effet d'affaiblissement à travers d'autres études empiriques qui sont, par exemple, construites sur la linguistique de corpus. De cette manière, il sera possible de saisir le phénomène à travers des angles d'évaluation et de production qui permettront de consolider les résultats présentés ici.

Nous voudrions remercier les locuteurs qui nous ont offert leurs jugements grammaticaux, grâce auxquels nous avons pu réaliser cette étude, ainsi que les collègues qui ont distribué le lien de notre sondage en ligne aux participants. Nous tenons également à remercier Annabelle Judel, assistante étudiante du deuxième auteur, pour son aide sur le formatage de la bibliographie et la numérotation. 
Nous voudrions remercier tout particulièrement Cédric Patin (Lille) pour ses remarques constructives pendant la préparation des énoncés test et pour la correction du français de cet article. Son soutien et sa disponibilité nous ont aidés énormément et ont permis un achèvement facile de notre article. Nous voudrions tout autant remercier Markus Bader et Yvonne Portele, membres de l'institut de psycholinguistique à Frankfurt, qui nous ont conseillé sur les questions liées à la structure et à la mise en œuvre de notre étude expérimentale. De surcroît, un merci tout particulier à Andreas Heuer pour l'aide qu'il nous a apportée pour l'analyse statistique descriptive. Toutes les erreurs restantes sont les nôtres. Notre étude a reçu un appui financier de l'Université Goethe de Frankfurt grâce au Prix d'excellence dans l'enseignement supérieur financé qui a été décerné au deuxième auteur en 2019.

\section{Références bibliographiques}

Aronoff, M. (1976). Word Formation in Generative Grammar. Cambridge: MIT Press.

Bouchard, D. (1982). On the Content of Empty Categories. Dordrecht: Foris.

Bouchard, D. (1983). The Avoid Pronoun Principle and the Elsewhere Principle. In: Sells, P. \& C. Jones (eds.), Proceedings of ALNE 13/NELS 13. Amherst (Mass.): GLSA, pp. 29-36.

Costantini, F. (2005). On Obviation in Subjunctive Clauses: The State of the Art. Annali Di Ca'Foscari. Università di Venezia XLIV(1-2): 97-132.

Costantini, F. (2009). Interface Perspectives on Clausal Complementation. The Case of Subjunctive Obviation. Venezia: Libreria Editrice Cafoscarina.

Costantini, F. (2013). Evidence for the competition-based analysis of subjunctive obviation from relative and adverbial clauses in Italian. In: Baauw, S., F. Drijkoningen, L. Meroni \& M. Pinto (eds.), Romance Languages and Linguistic Theory 2011: Selected papers from 'Going Romance' Utrecht 2011. Amsterdam: John Benjamins, pp. 75-92.

Costantini, F. (2016). Subject Obviation as a Semantic Failure: a Preliminary Account. Annali di Ca' Foscari 50: 109-131.

De Mulder, W. (2010). Mood in French. In: Rothstein, B. \& R. Thieroff (eds.), Mood in the Languages of Europe. Amsterdam: John Benjamins, pp. 157-178.

Farkas, D. (1992). On Obviation. In: Sag, I. \& A. Szabolcsi (eds.), Lexical Matters. Stanford: CSLI, pp. 85-109.

Featherston, S. (2006). Experimentell erhobene Grammatikalitätsurteile und ihre Bedeutung für die Syntaxtheorie. In: Kallmeyer W. \& G. Zifonun (eds.), Sprachkorpora - Datenmengen und Erkenntnisfortschritt. Berlin: de Gruyter, pp. 49-69.

Feldhausen, I. (2007). The Relation of Obviation and Control: Subjunctives and Infinitives in Spanish. Ms., ZAS Berlin.

Feldhausen, I. (2010). Sentential Form and Prosodic Structure of Catalan. Amsterdam: John Benjamins.

Kempchinsky, P. (1987). The Subjunctive Disjoint Reference Effect. In: Neidle, C. \& R. Nuñez Cedeño (eds.), Studies in Romance Languages. Dordrecht: Foris, pp. 123-140.

Kempchinsky, P. (2009). What can the subjunctive disjoint reference effect tell us about the subjunctive? Lingua 119(12): 1788-1810.

Le Goffic, P. (1993). Grammaire de la Phrase Française. Paris: Hachette Supérieur.

Luján, M. (1999). A Unified Approach to Control and Obviation. In: Franco, J., A. Landa \& J. Martín (eds.), Grammatical Analyses in Basque and Romance Linguistics. Amsterdam: John Benjamins, pp.105-130.

Manzini, M. (2000). Sentential Complementation. The Subjunctive. In: Coopmans, P., M. Everaert \& J. Grimshaw (eds.), Lexical Specification and Insertion. Amsterdam: J. Benjamins, pp. 241-268. 
Mosegaard Hansen, M. (2016). The Structure of Modern Standard French: A Student Grammar. Oxford: Oxford University Press.

Picallo, C. (1985). Opaque domains. Ph.D. Thesis, CUNY.

Poplack, S. (1992). The inherent variability of the French subjunctive. In: Laeufer, C. \& T. Morgan (eds.), Theoretical analyses in Romance linguistics, 235-263. Amsterdam: John Benjamins.

Quer, J. (2006). Subjunctives. In: Everaert, M. \& H. van Riemsdijk (eds.), The Syntax Companion, vol. IV. Oxford: Blackwell, pp. 660-684.

Quer, J. (2017). Subjunctives. In: Everaert, M. \& H. van Riemsdijk (eds.), The Blackwell Companion to Syntax, Second edition. Oxford: Blackwell (https://doi.org/10.1002/9781118358733 .wbsyncom113).

Raposo, E. (1985). Some Asymmetries in the Binding Theory in Romance. The Linguistic Review 5: $75-110$.

Riegel, M., J.-C. Pellat \& R. Rioul (20145). Grammaire méthodique du français. Paris : PUF.

Rizzi, L. (1991). On the anaphor-agreement effect. In: Rizzi, L. (ed.), Comparative Syntax and Language Acquisition. London/New York: Routledge, pp. 158-173.

Romero Mérida, R. (2013). Processing Obviation in Spanish. Master's Thesis, University of Utrecht, $<$ http://dspace.library.uu.nl/handle/1874/283715>.

Ruwet, N. (1991). Je veux partir / *Je veux que je parte: on the distribution of finite complements and infinitival complements in French. In: Ruwet, N. (1991). Syntax and Human Experience. The University of Chicago Press. [publié avant dans : Cahiers de Grammaire 7 (1984), p.75-138.]

Schlenker, P. (2005). The Lazy Frenchman's Approach to the Subjunctive. In: Geerts, T., I. van Ginneken \& H. Jacobs (eds.), Romance Languages and Linguistic Theory 2003. Amsterdam: John Benjamins, pp. 269-309.

Schütze, C. \& J. Sprouse. (2013). Judgment data. In: Podesva, R. \& D. Sharma (eds.), Research methods in linguistics. New York: Cambridge University Press, pp. 27-50.

Sprouse, J. (2018). Acceptability judgments and grammaticality, prospects and challenges. In: Hornstein, N., H. Lasnik, P. Patel-Grosz \& C. Yang (eds.), Syntactic Structures after 60 Years The Impact of the Chomskyan Revolution in Linguistics. Berlin: De Gruyter, pp. 195-224.

Suñer, M. (1986). On the Referential Properties of Embedded Finite Clause Subjects. In: Bordelois, I., H. Contreras \& K. Zagona (eds.), Generative Studies in Spanish Syntax. Dordrecht: Foris, pp. 183-196.

Szucsich, L. (2009). Obviation und temporale Abhängigkeit bei Subjunktiven. Zeitschrift für Slawistik 54(4): 398-415.

Zhang, N. (2010). Coordination in Syntax. Cambridge: CUP.

\footnotetext{
* Auteur correspondant : ingo.feldhausen[at]gmx.de, feldhausen[at]em.uni-frankfurt.de

i On notera que Poplack (1992: 237) a relevé des exemples de coréférence dans les entretiens qu'elle a conduits avec des locuteurs natifs du français québécois. Parce que nous nous focalisons ici sur le français européen, et parce que nous n'avons pas examiné les cas de variation de sélection du mode, nous ne pouvons qu'évoquer ses découvertes à ce stade.

ii L'idée principale des démarches fondées sur la théorie du liage est la suivante : l'obviation résulte d'une extension du domaine du liage de l'énoncé subordonné, si bien que le sujet de l'énoncé matrice fait partie du domaine et, en conséquence, la coréférence est exclue à cause de la condition B du liage (p.ex. Picallo 1985, Kempchinsky 1987, Manzini 2000). Les faiblesses de ces démarches sont décrites en détail dans Costantini (2009).

iii Nous sommes conscients du fait que les classes de verbes ne sont pas limitées aux verbes de volonté et de désir (voir, p. ex. Costantini 2009). Toutefois, parce que nos stimuli ne font intervenir que le verbe vouloir dans l'énoncé matrice, la généralisation de Farkas (1992) nous suffit.
} 